\title{
Well-Polarized Quantum-dot Cellular Automata Inverters
}

\author{
Keivan Navi \\ Nanotechnology and Quantum \\ Computing Lab. \\ G.C., Shahid Beheshti \\ University, Tehran, Iran
}

\author{
Mohammad A.Tehrani \\ Nanotechnology and Quantum \\ Computing Lab. \\ G.C., Shahid Beheshti \\ University, Tehran, Iran
}

\author{
Maliheh Khatami \\ Nanotechnology and Quantum \\ Computing Lab. \\ G.C., Shahid Beheshti \\ University, Tehran, Iran
}

\begin{abstract}
In past few years many efforts have been made on Quantumdot cellular automata (QCA) because it seems a good candidate for implementing next generation computers. Other technologies use electricity voltage or current to represent the binary values. However, in QCA it represents with charge polarization. In this paper two inverters are proposed whose polarizations are improved and their output signals are more robust. Consequently, the devices are more tolerable in noisy environment and they can perform more reliable. Finally the functionality of the models is verified by QCADesigner as a standard simulator for QCA models.
\end{abstract}

\section{Keywords}

Single Electron Devices, Quantum-dot Cellular Automata, Polarization, Inverter

\section{INTRODUCTION}

Moore's low predicts that the number of transistors in integrated circuits (IC) doubles every eighteen month. It means that, the technology of IC fabrication improves and compaction of IC's increases the performance of circuits [1].

In recent years, complementary metal-oxide semiconductor (CMOS) technology has faced physical limitation in feature sizing. It also has intolerable power consumption in high frequencies and could not afford the request for those frequencies at nano scale fabrication. Scaling-down CMOS integrated circuits is being aggressively followed by shrinking transistor dimension and reducing power supply voltage, despite the fact that these factors decrease the operational frequency $[1,2]$.

To overcome CMOS limitations, many efforts are being made on nano scale technologies and it is expected to achieve $10^{12}$ device $/ \mathrm{cm}^{2}$ compaction in ICs. There are various types of nano scale architectures that have been focused on, such as single electron transistor (SET), carbon nano-tube (CNT), and quantum-dot cellular automata (QCA) [3, 4].

One of the promising nanotechnologies is Quantum-dot cellular Automata which has brought together all the best features. It could make digital circuits with tremendously low feature size as low as molecular scale and ultra-high frequencies as high as $2 \mathrm{THz}$. It also has very low power consumption about $10^{-10}$ Watt per input signal that makes it a appropriate technology for the next generation circuits and a perfect candidate for CMOS replacement. With QCA's design, the problems associated with small output current or voltage and parasitic capacitance of wire between gates do not happen $[4,5]$.
It also presents a novel approach to digital logic for transmitting data and represents logical values that do not prevent compaction of ICs. The major idea of QCA cells is hidden in its structure that is the use of electron position in cells instead of voltage level or current amplitude. This phenomenon prevents any electrical current through the wire so the power consumption would be near zero [6]. Although it has many fascinating features, operating point temperature have to be kept near absolute zero. However many effort are being done to overcome the problem using molecular QCA to realize it in room temperature $[7,8,9]$.

The main concern of this article is to propose two novel designs for QCA inverter whose output signals are more polarized. Therefore, the signals can tolerate more noises and the circuit could be fabricated in higher temperature.

The next section provides a review of QCA theory and the operation of the "standard cell". In section three, the previous works for inverter are studied. In fourth section, the proposed models are introduced and prominences of these works are expressed. Finally in section five, simulation results are presented.

\section{QCA BASICS}

A typical QCA cell consists of four quantum dots located at the corners of a cell, whose two surplus electrons can freely tunnel between the four dots (Fig.1.a). The intercellular interaction is provided by the coulomb repulsion between electrons in each cell. The high barriers at cell borders suppress the electrons from tunneling out of the cell [6].

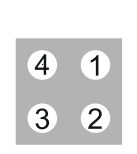

(a)

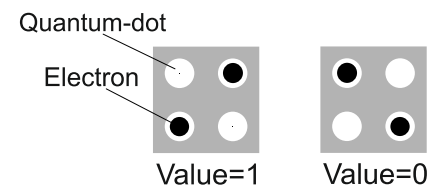

(b)
Fig 1. (a) A typical QCA cell, (b) Two charge configurations

Due to coulomb repulsion, electrons try to get as farther distance as possible, so they prefer to situate on diagonally opposite sites making the logical values 0 and 1 as is shown fig.1.b. The coulomb repulsion between electrons also exists between adjacent cells and it will be minimized when adjacent cells have the same charge configuration. Cell polarization $\mathrm{P}$ is defined as a quantifier for charge distribution among a cell as shown in (1). 
$P=\frac{\left(\rho_{1}+\rho_{3}\right)-\left(\rho_{2}+\rho_{4}\right)}{\rho_{1}+\rho_{3}+\rho_{2}+\rho_{4}}$

Where $\rho_{i}$ stands for electronic charge at site $i$. As a result of this equation when electrons are at site 1 and 3 the polarization is +1 and in the other state the polarization is -1 .

Arranging some cells in a line makes a wire which could transfer signal without any current flows into or out of the cells. Any given charge configuration at each terminal could be driven at the other side (Fig.2.a).

Besides, when each cell is rotated by $45^{\circ}$ from the base line, the signal inverse in every steps. Similarity or dissimilarity of the input and output depends on the number of cells through the wire (Fig.2.b). [10, 11]

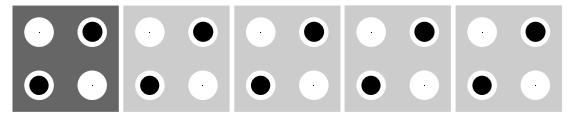

(a)

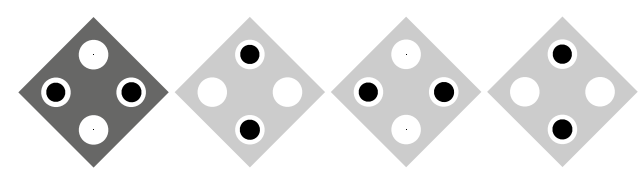

(b)

Fig. 2 (a) Standard wire (b) Rotated wire (It also known as chained-inverter)

\section{PREVIOUS WORKS}

One of the most important gates in QCA is inverter, because in every logic circuit it should be used. Hence constructing more efficient inverters is a great interest. The first QCA inverter was introduced by Lent et al. [6]. It simply inverts the signal by a cell displacement as is shown in Fig.3.a. The next model is very similar to first one and only input and output cells are on a straight line (Fig.3.b) [12]. In the third design illustrated in Fig.2.c the signal is divided into two paths. It amplifies the signal results in better polarization at output [13].

All introduced models are implemented in one layer and are worth for single layer design. However, for multi-layer circuits, more robust inverters could be utilized implemented in single or multiple layers.

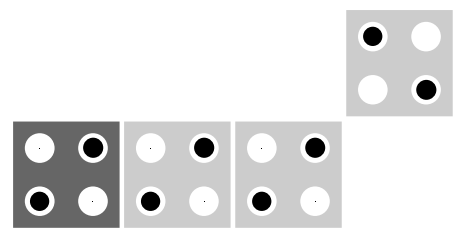

(a)

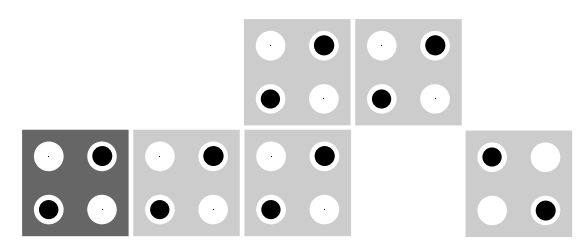

(b)

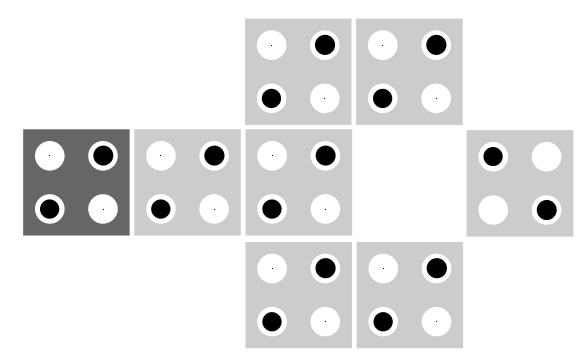

(c)

Fig. 3 (a, b, c) Several QCA inverters.

\section{PROPOSED DESIGNS}

When two QCA cell affect each other on a straight line, the signal propagates regularly. However, when they affect with a $45^{\circ}$ angle, the signal is inverted. This phenomenon is the basis of all surveyed QCA inverters. The proposed models are also used this idea with just a little change. In all the previous models, there is a $45^{\circ}$ angle between two adjacent cells placed in the same layer. However, in the new models, the neighbor cells do not located in the same layer and they inverse the signals while transferring from one layer to another.

The proposed models are shown in Fig.4.a and Fig.4.b. The first model looks like mentioned inverter in Fig.3.b and the second model is similar to Fig.3.c. However, they are implemented in two and three layers respectively.

Although polarizations in both models are better than their single layer counterparts, each model has its own cons and pros that the designer should select the best one according to the application. The polarization in second proposed model is better than the first one. However, the second model is implemented in three layers instead of two layers for the first one. 


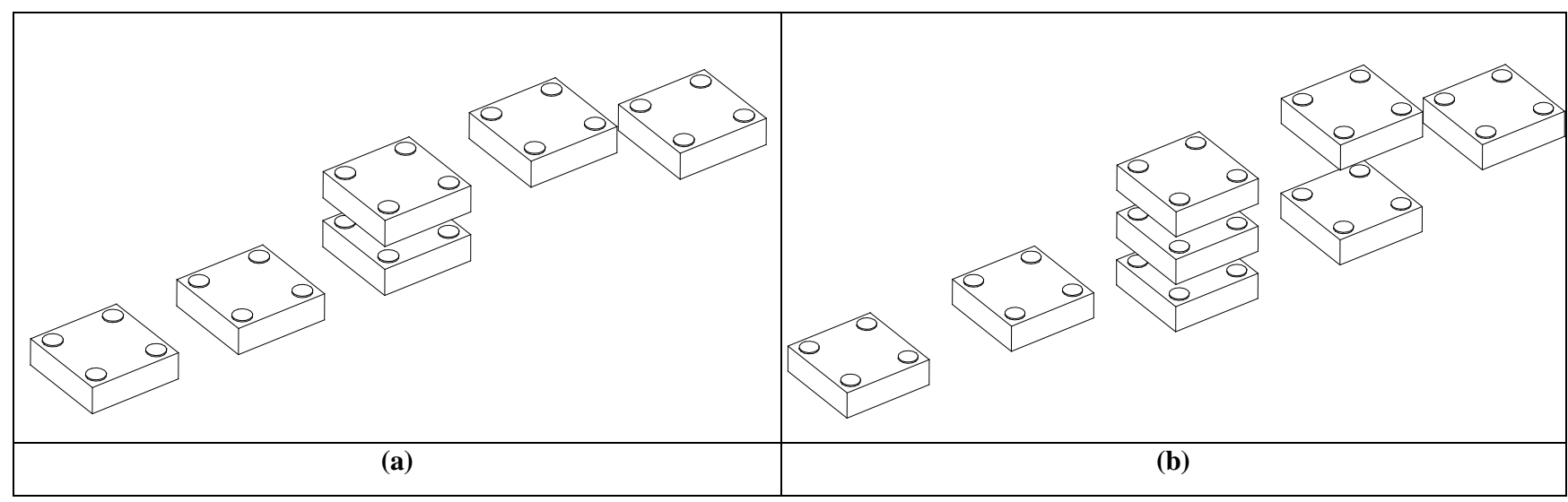

Fig. 4 (a) Proposed two-layer inverter. (b) Proposed three-layer inverter.

Table 1. The comparison results between proposed designs and previous circuits

\begin{tabular}{|c|c|c|c|c|c|}
\hline Model & $\begin{array}{l}\text { Lent et } \\
\text { al. [6] }\end{array}$ & $\begin{array}{l}\text { Farazkish } \\
\text { et al. [12] }\end{array}$ & $\begin{array}{l}\text { Proposed model } \\
\text { (Two-layer) }\end{array}$ & $\begin{array}{l}\text { Tougaw } \\
\text { et al. [13] }\end{array}$ & $\begin{array}{l}\text { Proposed model } \\
\text { (Three-layer) }\end{array}$ \\
\hline Complexity & 4 & \multicolumn{2}{|r|}{6} & \multicolumn{2}{|r|}{8} \\
\hline Polarization & 0.559 & 0.486 & 0.588 & 0.775 & 0.842 \\
\hline Polarization Increase Ratio & - & \multicolumn{2}{|r|}{$21.0 \%$} & \multicolumn{2}{|r|}{$8.6 \%$} \\
\hline Area $\left(\mathrm{nm}^{2}\right)$ & 2964 & 3724 & 1764 & 5684 & 1764 \\
\hline Area Decrease Ratio & - & \multicolumn{2}{|r|}{$52.6 \%$} & \multicolumn{2}{|r|}{$69.0 \%$} \\
\hline
\end{tabular}

\section{SIMULATION RESULTS}

Proving the functionality of proposed models, the QCADesigner is used because it is the most accurate computer aided design tool for QCA. It calculates the cell to cell responses according to the kink energy and tries to find the ground state of the model. There are two types of simulation models used for simulating: Coherence Vector and Bistable Approximation [14].

Coherence Vector model computes the time-dependent state of cells based on their king energy. The king energy is the energy cost of a systems produced by Coulombic repulsion between each pair of electrical charges. Every system likes to minimize its kink energy to achieve more stable state until it reaches to its ground state. The Coherence Vector model is used for dynamic behavior of cell's polarization exchanging so the accuracy of this model depends on timing step.

Bistable Approximation model computes time-independent state of cells based on the king energy between them. This model tries to gain the result for steady state of the system therefore it could reduce the total time of simulation. In the proposed models the paths between input and output are not so long and numbers of cells are few enough, so Coherence Vector model is used.
In QCADesigner, all cells are similar and the length of each cell is $18 \mathrm{~nm}$. The following parameters are used for a Bistable Approximation: Number of samples $=12800$, convergence tolerance $=0.001$, radius of effect $=65 \mathrm{~nm}$, relative permittivity $=12.9$ (for GaAs), clock high $=9.80 \mathrm{e}-$ $22 \mathrm{j}$, clock amplitude factor $=2$, layer separation $=11.5$, maximum iterations per sample $=100$.

Most of parameters are default values in QCADesigner where the calculations are at zero temperature. The comparison results between proposed designs and previous circuits are shown in Table 1 and the output signal performance is demonstrated in fig. 5 . 


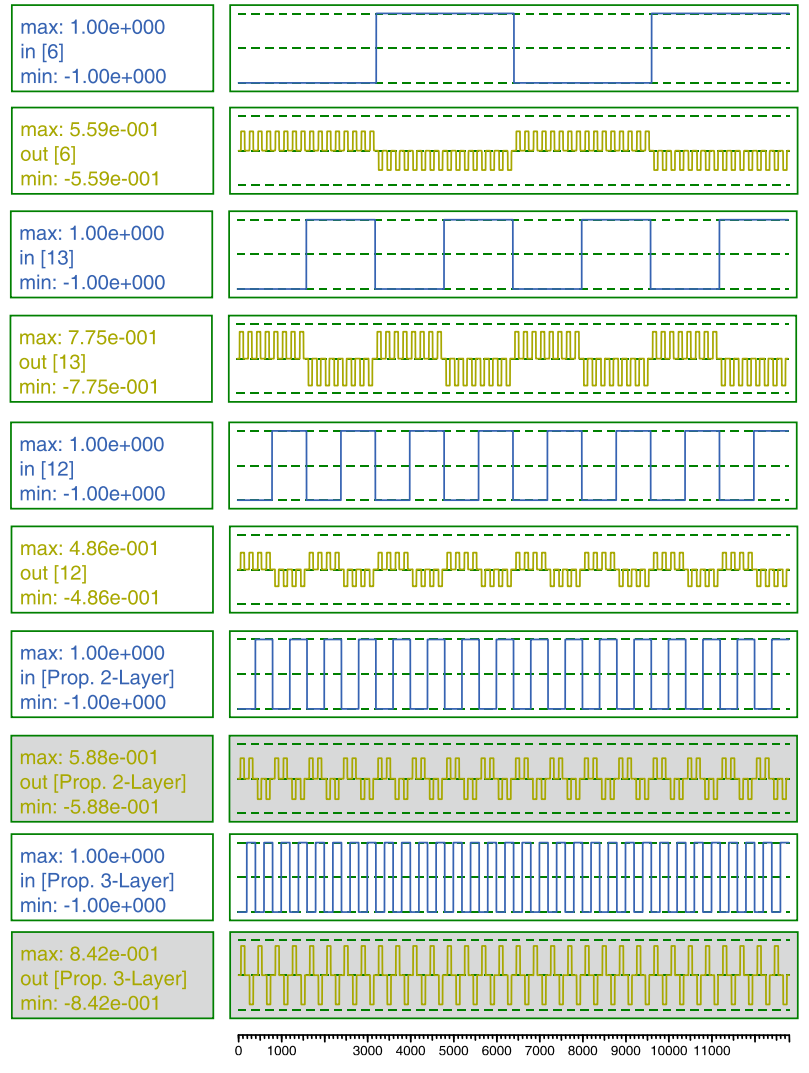

Fig. 5 Simulation Results

\section{CONCLUSION}

Quantum-dot Cellular Automata is a promising nanotechnology whose ultra-low power consumption and tremendous speed attracts many researchers to focus on. One of the basic elements of the QCA design is inverter that has been implemented using various methods. The proposed inverter models perform as fast as the old ones, besides the output is more polarized and signal is more noise tolerable. They also use significantly less area than the other models so in complex circuits with millions of inverters, it is more economic.

One of the design criteria is spacing between wires. Because the models' width in main cell layer is only one cell, wire spacing is easier and signal interfere is significantly reduced.

\section{REFERENCES}

[1] R.D. Isaac, "The future of CMOS technology", IBM Journal of Research and Development, Vol. 44, No. 3, 2000, pp. 369-378.

[2] J. Huang, M. Momenzadeh, and F. Lombardi, "An Overview of Nanoscale Devices and Circuits", IEEE
Design \& Test of Computers, Vol. 24, No. 4, 2007, pp. 304-311.

[3] F., Safaei, M.H. Moaiyeri, and M.A. Tehrani, "Design and Evaluating Carbon Nanotube Interconnects for a Generic Delta MIN", 19th Euromicro International Conference on Parallel, Distributed and Network-Based Processing, 2011.

[4] M.A. Tehrani, F. Safaei, M.H. Moaiyeri, and K. Navi, "Design and implementation of Multistage Interconnection Networks using Quantum-dot Cellular Automata", Elsevier Microelectronics Journal, Vol. 42, No. 6, 2011, pp. 913-922.

[5] K. Kim, K. Wu, and R. Karri, "Quantum Dot Cellular Automata Design Guideline", IEICE Transaction, Vol. E89-A, No. 6, 2006, 1607-1614.

[6] C.S. Lent, P.D. Tougaw, W. Porod, and G.H. Bernstein, "Quantum cellular automata", Nanotechnology, Vol. 4, No. 1, 1993, pp. 49-57.

[7] G.L. Snider, A.O. Orlov, I. Amlani, G.H. Bernstein, C.S. Lent, J.L. Merz, and W. Porod, "Experimental Demonstration of Quantum -dot Cellular Automata", IOP Semiconductor Science and Technology, Vol. 13, 1998, pp. A130-A134.

[8] C.S. Lent, "Bypassing the Transistor Paradigm", Science, Vol. 288, 2000, pp. 1597-1599.

[9] G. Bazan, A.O. Orlov, G. L. Snider, and G. H. Bernstein, "Charge detector realization for $\mathrm{AlGaAs} / \mathrm{GaAs}$ quantumdot cellular automata", Journal of Vacuum Science and Technology, Vol. 14, No. 6, 1996, 4046-4050.

[10] C.S. Lent, and P.D. Tougaw, "A Device Architecture for Computing with Quantum Dots", Proceeding of the IEEE, Vol. 85, No. 4, 1997, pp. 541-557.

[11] C.S. Lent, P.D. Tougaw, and W. Porod, "Quantum cellular automata: the physics of computing with arrays of quantum dot molecules", Proc. PhysComp'94, 1994, pp. 5-13.

[12] R. Farazkish, M.R. Azghadi, K. Navi, and M. Haghparast, "New Method for Decreasing The Number of Quantum Dot Cells in QCA Circuits", World Applied Sciences Journal, Vol. 4, No. 6, 2008, pp. 793-802.

[13] P.D. Tougaw, and C.S. Lent, "Logical devices implemented using quantum cellular automata", Journal of Applied Physics, Vol. 75, No. 3, 1993, pp. 1818-1825.

[14] K. Walus, T.J. Dysart, G.A. Jullien, and R.A. Budiman, "QCADesigner: a rapid design and Simulation tool for quantum-dot cellular automata", IEEE Transactions on Nanotechnology, Vol. 3, No 1, 2004, pp. 26-31. 